Received: 16.08 .2020

Revised: 24.09 .2020

Accepted: 16.10 .2020

DOI: $10.17804 / 2410-9908.2020 .5 .045-057$

\title{
APPLYING AN UPDATED METHOD OF MARKERS TO DEFINING TRANSIENT FORCE IMPACT UNDER MULTIPHASE FLOWING
}

\author{
V. I. Pegova)* and I. Yu. Moshkin ${ }^{\text {b) }}$ \\ South Urals Federal Research Center of Mineralogy and Geoecology, \\ Ural Branch of the Russian Academy of Sciences, Ilmen State Nature Reserve, \\ Bldg. 1, Miass, 456300, Russian Federation \\ Academician V. P. Makeyev State Rocket Centre JSC, \\ 1 Turgoyakskoye Hwy., Miass, 456300, Russian Federation \\ a) ofpat@mail.ru; \\ b) moshkin_igor@mail.ru \\ *Corresponding author. E-mail: ofpat@mail.ru \\ Address for correspondence: pr. Oktyabrya, 16, Room 103, OFPAT, 456318, Miass, Russian Federation \\ Tel.: +7 (351) 3288550
}

Numerical simulation of transient hydrodynamic forces from shaped gas cavities formed in liquid under active interaction of liquid and a jet source of high-temperature gas and intensive heat and mass transfer is performed. To solve the task, a method of coarse particle markers with, as opposed to the classical one, an additional stage, when moving boundaries of different media in cells with interfaces of these media are as if stitched, is updated. In addition, problems of inter-media heat and mass transfer by condensation and evaporation are simultaneously solved. The predicted results are compared with the experimental data. Validation and verification are performed by comparing the analysis results with the experimental data. The applicability of the updated method of coarse particle markers to defining transient force impact under multiphase flowing is demonstrated.

Keywords: heat and mass transfer, two-phase flow, method of markers, transient impact, multiphase flow.

\section{Acknowledgment}

The work was performed with the support from the South Urals Federal Research Center of Mineralogy and Geoecology of the Ural Branch of the Russian Academy of Sciences and the Academician V. P. Makeyev State Rocket Centre JSC under the state assignment to the South Urals Federal Research Center of Mineralogy and Geoecology of the Ural Branch of RAS, theme No. 0431-2014-0001.

\section{References}

1. Degtyar V.G., Pegov V.I. Gidrodinamika podvodnogo starta raket [Hydrodynamics of Underwater Missile Launching]. Moscow, Mashinostroenie Publ., 2009, 448 p. (In Russian).

2. Degtiar V.G., Pegov V.I., Moshkin I.Yu., Cheshko A.D. Mathematical modeling of the processes of heat and mass transfer of hot gas jets with fluid during underwater rocket launches. Teplofizika Vysokikh Temperatur, 2019, vol. 57, iss. 5, pp. 742- 747. DOI: 10.1134/S004036441905003X. (In Russian).

3. Pegov V.I., Moshkin I.Yu. Dynamic analysis of a ballistic missile model. Vestnik YuUrGU. Ser. Mat. Model. Progr., 2017, vol. 10, iss. 4, pp. 56-63. DOI: 10.14529/mmp170406. (In Russian). 4. Degtiar V.G. Pegov V.I., Cheshko A.D. Investigation of ignition of a submerged jet engine. Nauchno-technicheskiy Vestnik Povolzhya. - 2016. - No. 5. - P. 181-189. (In Russian). 
5. Pegov V.I., Cheshko A.D., Moshkin I.Yu., Merkulov Ye.S. Experimental modeling and simulation of launching effect on a submarine. In: A look into the future-2016: proceedings of XIV Youth Scientific and Technical Conference, Saint Petersburg, CDB ME "Rubin" Publ., 2016, pp. 598-605. (In Russian).

6. Pegov V.I., Moshkin I.Yu. Analysis of fluid dynamics of cavitational launch technique Chelyabinsk Physical and Mathematical Journal, 2018, vol. 3, iss. 4, pp. 476-485. DOI: 10.24411/2500-01012018-13408. (In Russian).

7. Pegov V.I., Moshkin I.Yu., Merkulov Ye.S., Cheshko A.B. Numerical simulation of hydrodynamic loads on starting rocket and submarine, Bulletin of Almaz-Antey JSC, 2016, no. 3 (18), pp. 65-70. (In Russian).

8. Degtiar V.G., Moshkin I.Yu., Pegov V.I., Kalashnikov S.T., Khlybov V.I. $R U$ CNC_RNSG 2015613842 (08 Sept. 2014). (In Russian).

9. Kostin G.F., Degtiar V.G., Kalashnikov S.T., Savelyev V.N., Tyumentsev V.A., Khlybov V.I., Shvaleva R.K. Experimental-analytical methodology used to define parameters of sandy-grained roughness on burned surfaces of composite thermal-protective materials. Konsruktsii iz Kompozitsionnykh Materialov, 2018, no. 3, pp. 29-39. (In Russian).

10. Kostin G.F., Zakharyevich D.A., Kalashnikov S.T., Savelyev V.N., Shvaleva R.K. Methodology and results of evaluation of variations in thermal and physical characteristics of a carbon fiber composite on the basis of a phenol-formaldehyde binding agent under heating and decomposition. Konsruktsii iz Kompozitsionnykh Materialov, 2018, no. 4, pp. 63-70. (In Russian).

11. Kalashnikov S.T., Mokin Yu.A., Shvaleva R.K. Design and experimental study of changes in the shape of small lateral surface distortions during ablation of carbon-composite thermal protection of the reentry vehicle model. Konsruktsii iz Kompozitsionnykh Materialov, 2018, no. 4 (152), pp. 42-47. (In Russian).

12. Mokin Yu.A., Kalashnikov S.T., Shvaleva R.K. Estimate of variance of disturbance aerodynamic normal force coefficient of rotary body with small irregular surface distortions of composite thermal protection coating at supersonic flow. Konsruktsii iz Kompozitsionnykh Materialov, 2020, no. 3, pp. 3-8. (In Russian). 
Подана в журнал: 16.08 .2020

УДК 629.76.085.5:532.5:519.673

DOI: $10.17804 / 2410-9908.2020 .5 .045-057$

\title{
ПРИМЕНЕНИЕ МОДЕРНИЗИРОВАННОГО МЕТОДА МАРКЕРОВ ДЛЯ ОПРЕДЕЛЕНИЯ НЕСТАЦИОНАРНОГО СИЛОВОГО ВОЗДЕЙСТВИЯ ПРИ МНОГОФАЗНОМ ОБТЕКАНИИ
}

\author{
В. И. Пегов ${ }^{\mathrm{a})^{*}}$, И. Ю. Мошкин ${ }^{\text {) }}$ \\ Южно-Уральский федеральный научный центр минералогии и геоэкологии \\ Уральского отделения Российской академии наук, \\ к. 1, Ильменский заповедник, 456300, г. Миасс, Российская Федераиия \\ AO «Государственный ракетный иентр имени академика В.П. Макеева», \\ 1, Тургоякское шоссе, 456300, г. Миасс, Российская Федераичия \\ а) $\otimes$ ofpat@mail.ru;
б) moshkin_igor@mail.ru \\ *Ответственный автор. Электронная почта: ofpat@mail.ru \\ Адрес для переписки: пр. Октября, 16, к. 103, ОФПАТ, 456318, г. Миасс, Российская Федерация \\ Тел.: +7 (351) 328-85-50
}

Проведено численное имитационное моделирование нестационарных гидродинамических сил от газовых полостей сложной формы, образующихся в жидкости при активном взаимодействии жидкости и струйного источника высокотемпературного газа при интенсивном тепломассообмене. Для решения данной задачи был модернизирован метод маркеров крупных частиц, когда, в отличие от классического, вводится дополнительный этап, на котором происходит «сшивка» подвижных границ различных сред в ячейках, где проходит раздел этих сред. При этом одновременно решаются задачи тепломассообмена между средами, конденсацией и испарением. Результаты расчетов сравниваются с экспериментальными данными. Валидация и верификация проведены путем сравнения результатов расчетов с опытными данными. Показана возможность использования модернизированного метода маркеров крупных частиц для определения нестационарных гидродинамических сил при многофазном обтекании.

Ключевые слова: тепломассообмен, двухфазное течение, метод маркеров, нестационарное воздействие, многофазное обтекание.

\section{1. Введение}

Выход автономного подводного аппарата, предназначенного для проведения научноисследовательских и спасательных работ, из транспортного контейнера осуществляется работой донного газогенератора, который создает на днище контейнера газовую область с повышенным давлением и температурой. При выходе аппарата из контейнера (раскупорке) происходит истечение сгенерируемого высокотемпературного газа в жидкость. В результате возникает сложная, существенно нестационарная картина взаимодействия горячих струй газа с водой. В течение многих лет ГРЦ Макеева проводит расчетно-теоретические и экспериментальные исследования подводного старта аппаратов и сопутствующих ему процессов, в частности со струйным истечением высокотемпературных газовых струй в жидкость [1-7].

В практике проектирования рассматривается широкий круг возможных схем старта. Отметим основные особенности различных схем с точки зрения процессов газодинамики и тепломассообмена. Так, если старт подводного аппарата осуществляется с применением аккумулятора давления и запуском двигательной установки после выхода из контейнера, то 
движение аппарата в контейнере будет происходить только за счет сил давления. В случае применения стартовых двигателей или маршевого двигателя необходимо учитывать тягу и ее изменение от противодавления. Тип источника продуктов сгорания, его расположение, схема подачи рабочего газа, химический состав продуктов сгорания будут также влиять на газодинамические и тепловые параметры.

Другим определяющим фактором является схема расположения амортизации аппарата. Пояса горизонтальной амортизации могут располагаться на аппарате или контейнере. Газодинамические и тепловые процессы в минометной схеме старта существенным образом зависят от обводов поясов амортизации, их расположения, степени обтюрации кольцевого зазора. В соответствии с этими особенностями принимается та или иная схематизация течения. Влияние вертикальной амортизации выражается посредством упругих сил на пути, соизмеримом с величиной деформации элементов вертикальной амортизации. В пусковых установках применяются также различные устройства удержания, фиксирующие аппарат на пусковом столе до достижения определенной величины поршневой силы. При «сухом» способе старта с подводного носителя для герметизации верхнего среза контейнера применяется жесткая или эластичная мембрана; известны также устройства, обеспечивающие равномерное открытие контейнера посредством хода аппарата. Процессы раскрытия верхнего среза контейнера и формирования газовой полости у верхнего среза контейнера при подводном старте существенным образом влияют на газодинамические параметры в свободные объемы контейнера. Кроме того, для уменьшения гидродинамических нагрузок на аппарат при старте с движущегося носителя могут применяться различные устройства, обеспечивающие двухфазное обтекание аппарата на подводном участке траектории, например газогенераторы наддува газовой каверны. Эти особенности обтекания оказывают влияние на изменение давления, как на корпус аппарата, так и в стенки контейнера, что требуется учитывать при расчете гидродинамических нагрузок и кинематических параметров движения аппарата при выходе из контейнера. Более плотная компоновка аппарата и элементов стартовой системы в ограниченном объеме контейнера носителя, усложнение геометрии трактов приводят к локальным неравномерностям газодинамического и теплового воздействия.

Цель работы - разработка метода определения силового воздействия на подводный аппарат с учетом нестационарного силового воздействия от многофазной среды с учетом тепломассобмена между различными компонентами среды при отделении от носителя. Для учета тепловых процессов проведена модернизация метода маркеров крупных частиц; разработан программный комплекс имитационного моделирования тепловых процессов при многофазном обтекании. Проводится сравнение расчетов с экспериментальными данными.

\section{2. Материал и методика}

Для решения задачи о движении границ многофазных сред сейчас существует множество различных численных методов, недостатком которых является недостаточная точность при решении задачи тепломассообмена при протекании взаимодействий в высокотемпературных многофазных газовых потоках. Для решения задачи о движении границы раздела двух сред «жидкость-газ» был модернизирован так называемый метод маркеров SMAC (Simplified Marker-and-Cell Method). (Класический метод позволяет проводить расчеты многофазной среды без учета тепломассообмена). Основная идея предлагаемого метода заключается в расщеплении каждого временного шага на два этапа:

1) на первом этапе проводится сквозной счет параметров состояния жидкости и газа независимо друг от друга, с одновременным рассмотрением маркеров частиц жидкости и газа, перемещающихся со скоростью своих сред, что позволяет определить скорость и положение подвижных границ этих сред на этом промежутке времени; 
2) на втором этапе происходит «сшивка» подвижных границ двух сред в ячейках, где проходит граница раздела этих сред, при этом одновременно решается задача тепломассообмена между жидкостью и газом.

Для первого этапа используется стандартная система уравнений SMAC - метода, на втором этапе расчета рассматривается двухфазная среда.

1. Уравнение состояния для воды:

$$
e_{1}=\frac{P_{1}-C_{0}^{2}\left(\bar{\rho}_{1}-\rho_{0}\right)}{\left(\gamma_{1}-1\right) \bar{\rho}_{1}},
$$

где скорость звука в воде $C_{0}=1515$ м/с; $\gamma_{1}=5,6 ; \rho_{0}=1020$ кг $/ \mathrm{M}^{3} ; \bar{\rho}_{1}-$ среднеобъемная плотность жидкости (воды).

2. Газ с уравнением состояния:

$$
e_{2}=\frac{P_{2}}{\left(\gamma_{2}-1\right) \bar{\rho}_{2}},
$$

где $\gamma_{2}=5,6$ - показатель адиабаты газа; $\bar{\rho}_{2}-$ среднеобъемная плотность газа.

В элементарном объеме $\mathrm{V}$ может находиться одновременно и та, и другая среда, занимая объемы $\mathrm{V}_{1}$ и $\mathrm{V}_{2}$ соответственно. Объемную долю газа в ячейке будем характеризовать величиной $\xi=\mathrm{V}_{2} / \mathrm{V}_{1}$, а $\rho_{1}$ и $\rho_{2}-$ истинные плотности среды; $\rho_{1}=\bar{\rho}_{1}(1-\xi)-$ плотность жидкости внутри общего объема V; $\rho_{2}=\bar{\rho}_{2} \xi$ - плотность газа внутри общего объема V; $\rho=\rho_{1}+\rho_{2}-$ общая плотность.

Законы сохранения запишем для осесимметричного случая в цилиндрической системе координат в следующем виде:

- уравнение массы для общей плотности среды:

$$
\frac{\partial \rho}{\partial t}+\operatorname{div}(\rho W)=0
$$

- уравнение массы для газа:

$$
\frac{\partial \rho_{2}}{\partial t}+\operatorname{div}\left(\rho_{2} W\right)=M
$$

- уравнение импульсов для общей среды:

$$
\frac{\partial \rho u}{\partial t}+\operatorname{div}(\rho u W)+\frac{\partial P}{\partial r}=0, \frac{\partial \rho v}{\partial t}+\operatorname{div}(\rho v W)+\frac{\partial P}{\partial z}=0,
$$

- уравнение энергии для общей среды:

$$
\frac{\partial\left(e+\rho \frac{W^{2}}{2}\right)}{\partial t}+\operatorname{div}\left[\left(e+\rho \frac{W^{2}}{2}\right) W\right]+\operatorname{div}(P W)=0,
$$


- уравнение энергии для газа:

$$
\frac{\partial\left(e_{2}+\rho_{2} \frac{W^{2}}{2}\right)}{\partial t}+d i v\left[\left(e_{2}+\rho_{2} \frac{W^{2}}{2}\right) W\right]+\frac{\rho_{2}}{\rho} W \cdot g r a d P+K P_{2} \operatorname{div} W=0 .
$$

Здесь $W$ - вектор скорости; $u, v$ - составляющие $W$ вдоль z и r соответственно; $M$ - отнесенная к единице объема быстрота прироста массы в данной ячейке за счет парообразования.

Слагаемое $P \operatorname{div} W$ в уравнении энергии определяет работу, совершенную над единичным объемом общей среды за единицу времени, а $K$ - долю этой работы, приходящейся на газ.

Для определения коэффициента $K$ необходимо найти работу, совершаемую при сжатии той и другой среды от давления $P-d P$ до давления $P$. Эта работа с учетом адиабатичности определяется следующим образом:

$$
d A_{1}=\frac{V(1-\xi) P_{1}}{\gamma_{1}\left(P_{1}-P_{0}\right)} d P ; d A_{2}=\frac{V \xi}{\gamma_{2}} d P ; P_{0}=\frac{\rho_{0} C_{0}^{2}}{\gamma_{1}},
$$

а долю работы $d A_{2}$ в общей работе $d A=d A_{1}+d A_{2}$ через коэффициент $K=\frac{d A_{2}}{d A}$ можно определить следующим образом:

$$
K=\frac{\xi \gamma_{1}\left(P+P_{0}\right)}{\xi\left(P+P_{0}\right) \gamma_{1}+(1-\xi) P \gamma_{2}}
$$

Для улучшения устойчивости счета первого этапа в уравнениях (3) и (4) вместо $P$ используем $P+q$, где $q$ - искусственное вязкостное давление, которое определим следующим образом:

$$
q=-\mu C_{0}^{2} h \frac{\partial u}{\partial r} \text { при } \frac{\partial u}{\partial r}<0, q=0 \text { при } \frac{\partial u}{\partial r} \geq 0,
$$

где $h$ - размер ячейки; $\mu$ - искусственная вязкость.

В системе уравнений (1)-(5) нет уравнения для определения объемной доли газа. Кроме того, из уравнений состояния (1) и (2) давление может быть определено только в случае заполнения ячейки одной средой: либо газом $(\xi=1)$, либо жидкостью $(\xi=0)$.

Для определения давления в смешанных ячейках, т. е. заполненных жидкостью и газом, предложена следующая схема. Термодинамическая система «газ-жидкость» в момент поступления газа в объем, заполненный жидкостью и газом, является термодинамически неравновесной, так как газ - среда более энергоемкая, чем жидкость. Установление термодинамического равновесия происходит в результате обмена внутренней энергией между газом и жидкостью внутри одной ячейки. Это происходит за счет работы сжатия сред. При этом суммарная внутренняя энергия ячейки не изменяется, а давление устанавливается одинаковое в жидкости и газе. Сжатие на первом этапе будем считать адиабатическим, т. е. можно записать:

$$
e_{1}+e_{2}=\operatorname{const}(\xi) ; P_{2} \xi^{\gamma_{2}}=\text { const }
$$

Учитывая все это, получим дифференциальное уравнение для давления в жидкости $P_{1}$ : 


$$
\frac{d P_{1}}{d \xi}=\frac{P_{1}+\rho_{0} C_{0}^{2}+P_{2}\left(\gamma_{1}-1\right)}{1-\xi} ; \xi \in[0,1]
$$

а давление газа $P_{2}$ определяется из закона адиабатического сжатия:

$$
P_{2}(\xi)=P_{2}\left(\xi^{n}\right)\left(\frac{\xi^{n}}{\xi^{n+1}}\right)^{\gamma_{2}} ; \xi \in[0,1],
$$

где $\xi^{n}$ - объемная доля в ячейке до перераспределения энергий в $n$ - временном слое.

Уравнение (7) будем интегрировать методом Рунге-Кутта на интервале $\left[\xi^{n}, \xi^{n+1}\right]$. Значение объемной доли $\xi^{n+1}$ определяем из условия:

$$
P_{1}\left(\xi^{n+1}\right)=P_{2}\left(\xi^{n+1}\right)
$$

Таким образом, решаем (7) с учетом (8) при следующих начальных условиях:

$$
P_{1}\left(\xi^{n}\right)=\frac{e_{1}\left(\gamma_{1}-1\right)+C_{0}^{2}\left[\rho_{1}-\rho_{0}\left(1-\xi^{n}\right)\right]}{1-\xi^{n}} \rho_{1} ; \quad P_{2}\left(\xi^{n}\right)=\frac{e_{2}\left(\gamma_{2}-1\right)}{\xi^{n}} \rho_{2} .
$$

В результате получаем объемную долю газа и давление в смешанной ячейке. На следующем шаге, исходя из особенностей постановки задачи, определяется изменение температуры газа в смешанных ячейках за счет тепломассообмена между газом и жидкостью, а также за счет фазовых переходов. При этом считается, в отличие от первого шага, что эти процессы носят изохорический характер, т. е. в этом случае газ не совершает работы и его объемная доля не изменяется, а меняется только его температура и давление, а следовательно, граница раздела двух сред сохраняется.

Для определения потери энергии при фазовом переходе воспользуемся следующим уравнением:

$$
\frac{d P}{d T}=\frac{h_{2}-h_{1}}{T\left(V_{2}-V_{1}\right)}
$$

где $h_{2}-h_{1}-$ представляет собой разность энтальпий сосуществующих фаз. Эта разность энтальпий является теплотой фазового перехода, т. е. теплотой, которая поглощается (или выделяется) веществом в процессе фазового перехода; $V_{1}$ - удельный объем жидкости на линии раздела, $V_{2}$ - удельный объем пара на линии раздела.

В расчетах теплоту парообразования определяем из следующего равенства:

$$
r_{0}=a-b T
$$

где $a$ есть величина постоянная для рассматриваемого газа, $b$ - коэффициент, который найдем из соотношения:

$$
\ln \frac{P_{S}}{P_{S}\left(T_{S}\right)}=\frac{a}{R}\left(\frac{1}{T_{S}}-\frac{1}{T}\right)+\frac{b}{R} \ln \frac{T_{S}}{T} .
$$

Здесь $P_{S}$ есть давление насыщения при температуре $T_{S}$. 
По вышеизложенному методу была составлена программа на ЭВМ, с помощью которой были проведены расчеты динамики всплывающих газовых полостей, заполненных горячим или холодным газом. При этом начальное давление газов отличалось от давления окружающей жидкости. Расчеты позволили проследить динамику свободных границ газовых полостей в жидкости, их деформацию вплоть до разрушения (распада). Данный программный комплекс позволяет также проводить имитационное моделирование движения и тепломассообмена многофазной среды в контейнере при выходе из него аппарата.

\section{3. Результаты и обсуждение}

Полученная система уравнений (раздел 2) была реализована в программном комплексе CNC_RGSKK[8], разработанным Южно-Уральским федеральным научным центром минералогии и геоэкологии УрО РАН совместно с АО «Государственный ракетный центр имени академика В.П. Макеева» в рамках государственного задания ЮУ ФНЦ МиГ УрО РАН по теме № 0431-2014-0001. Программный комплекс зарегистрирован в Роспатенте. Для подтверждения работоспособности модернизированного метода маркеров крупных частиц, а также созданного на его основе программного комплекса CNC_RGSKK, полученных результатов имитационного моделирования и проверки силового воздействия на аппарат были проведены экспериментальные исследования с использованием пространственной модели $(\mathrm{M}=1: 10)$ на установке (рис. 1), состоящей из форкамеры 1 , в которую поступал воздух из магистрали высокого давления, модели контейнера 2 и модели аппарата 3 с установленными на нем двумя поясами амортизации 4 и одним поясом обтюрации 5 , расположенным в нижней части аппарата.

Необходимое противодавление на выходе из контейнера создавалось дополнительным поддувом газов в емкость, окружающую модель аппарата и контейнера.

Методика эксперимента строилась в предположении квазистационарности процессов. Варьировались следующие параметры:

- положение аппарата относительно контейнера;

- степень обтюрации кольцевого зазора;

- давление в форкамере;

- давление среды на выходе из контейнера.

Отношение давления на выходе из кольцевого зазора к давлению в задонном объеме $\bar{P}=\frac{P_{H}}{P_{1}}$ составляло $0,2 \ldots 0,66$. На модель аппарата наносился визуализационный состав. В процессе эксперимента при выходе на стационарный режим замерялось давление в 30-40 точках на аппарате и контейнере.

На рис. 2 представлена типичная фотография реализуемой в кольцевом зазоре картины течения при прохождении аппаратом безразмерного пути 0,08 , когда зона равномерного течения отсутствует. При всех исследуемых режимах в плоскостях I и III (напротив широких протоков) отчетливо видны интенсивные струйные течения на всем промежутке между первым и вторым поясами амортизаторов. В плоскостях II и IV реализуется сложное вихревое течение.

При увеличении степени обтюрации струйные течения становятся более отчетливыми, при этом, чем больше перепад давления между входным и выходным сечениями кольцевого зазора, тем выше по потоку перемещается система вихревых и возвратных течений (плоскости II и IV на рис. 2).

В верхнем поясе амортизаторов поток перемешивается, и течение становится близким к равномерному. Анализируя полученные экспериментальные результаты по картине течения газов в кольцевом зазоре, можно сделать вывод, что картина течения в основном определяется конструктивным заполнением кольцевого зазора, т.е. геометрическими факторами, и 
слабо зависит от величины внешнего противодавления. Неравномерность проходных сечений вдоль окружности зазора между аппаратом и контейнером оказывает значительное влияние на характер течения.

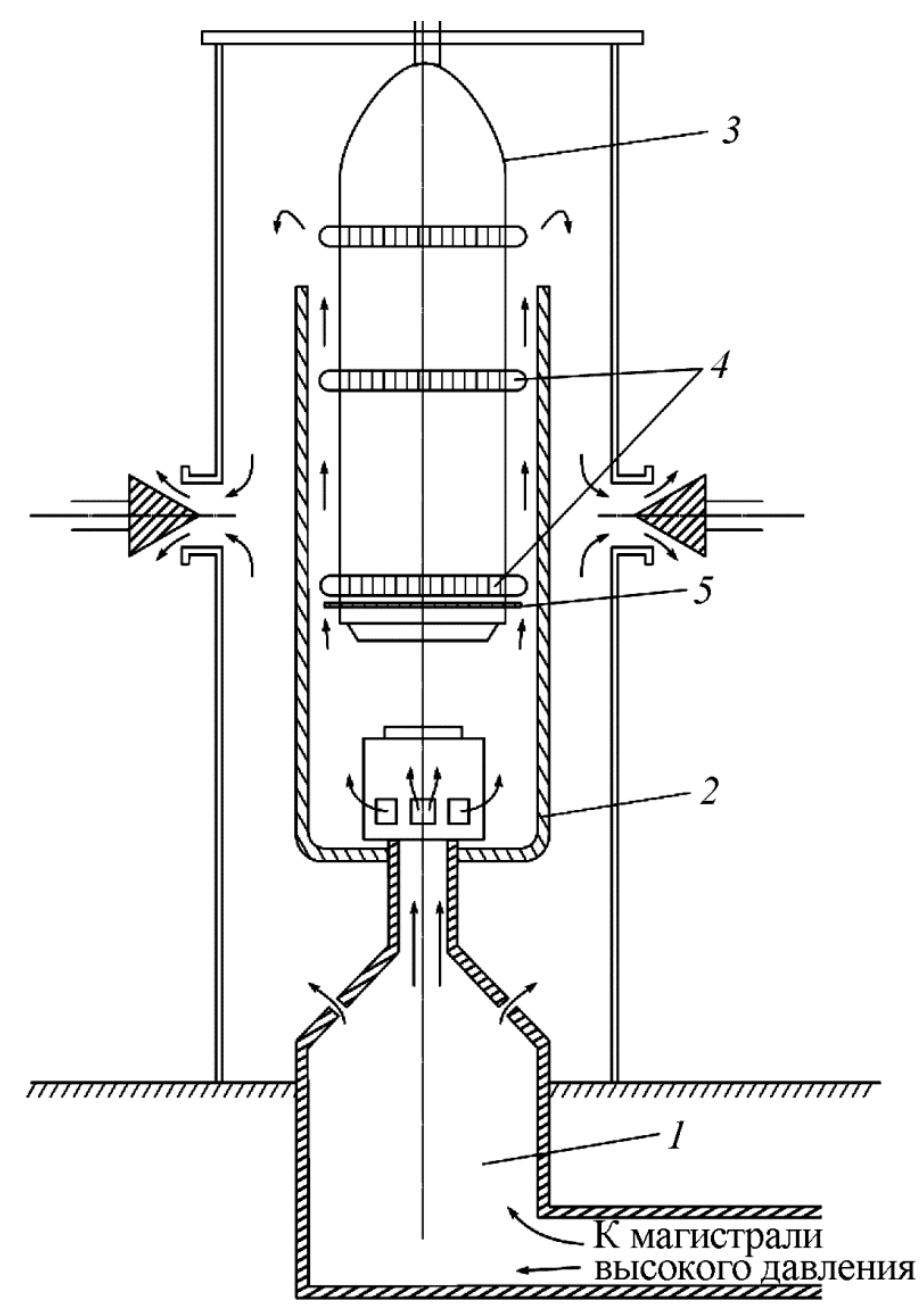

Рис. 1. Схема экспериментальной установки

Немаловажным вопросом, особенно при расчетах процесса теплообмена в задонном объеме, является изучение картины течения, зависящей как от используемых стартовых устройств, так и от положения аппарата в контейнере. В экспериментах изучалась картина течения, реализуемая для случаев старта аппарата с помощью односоплового и многосоплового двигателя первой ступени и старта с использованием акамулятора давления. Для последнего случая исследовалось влияние некоторых конструктивных особенностей (различные отражательные козырьки и щитки) на газодинамическую картину течения. Отношение диаметра аппарата к диаметру контейнера во всех экспериментах составляло 0,9. В результате проведенных экспериментальных исследований выявлено, что в задонном объеме картина течения зависит от положения аппарата в контейнере и характеризуется глубиной проникновения струй. При старте с помощью многосоплового стартового двигателя картина течения несколько усложняется. Дополнительно в центральной части днища контейнера возникают участки стекания направленных навстречу друг другу течений, формирующих отраженный в сторону днища аппарата поток, оканчивающийся вихревыми течениями.

При выходе аппарата из контейнера газы из кольцевого зазора поступают в объем газовой полости, однако наиболее интенсивный ее рост происходит после прохождения аппаратом пути, равного его длине, когда цилиндрическая часть аппарата проходит обтюрирующий пояс. 
В это время давление газов в контейнере, как правило, превышает давление окружающей среды, и газ из контейнера начинает перетекать в газовую полость. Вследствие большого массового расхода газов давление в полости возрастает и становится больше гидростатического, свободная граница полости приобретает значительные скорости по нормали. При выходе кормы аппарата из полости часть газов захватывается изделием и за его кормой образуется донная газовая каверна, которая имеет форму, близкую к цилиндрической. По мере роста газовых объемов давление газов в них падает и становится меньше гидростатического. Вследствие разрежения объем газовой полости начинает уменьшаться и в некоторый момент времени происходит полное схлопывание, объем донной газовой каверны полностью отделяется от объема контейнера. Валидация и верификация разработанного математического моделирования и программного комплекса проведены для двух случаев: при выходе кормы аппарата из контейнера и при продуве заполненного водой контейнера горячей струей газа.

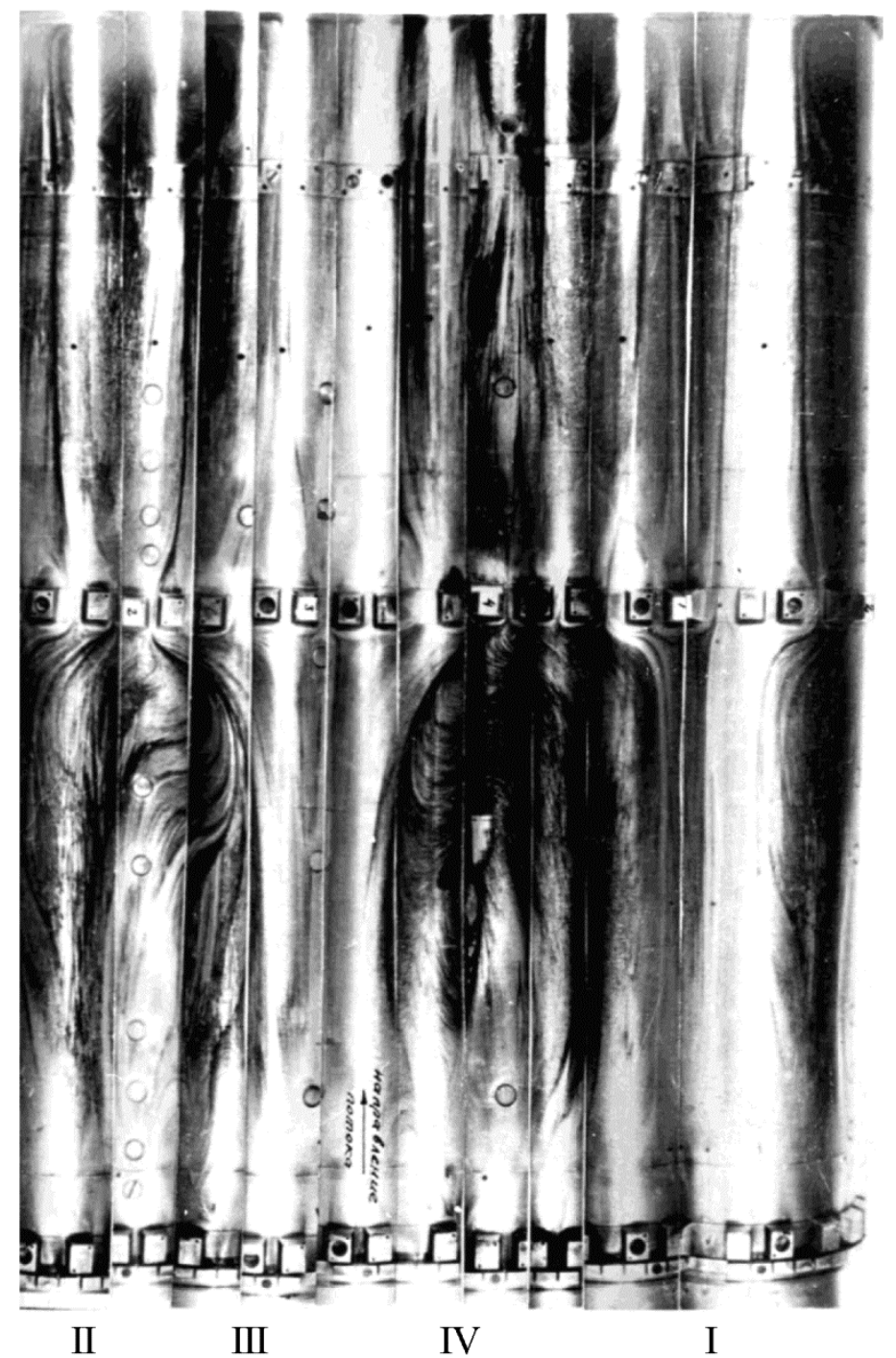

Рис. 2. Картина течения газа в кольцевом зазоре $\bar{P}=0,52 ; \bar{S}_{\text {обт, } 1}=80 \% ; \bar{S}_{\text {обт, } 2}=56 \%$

На рис. 3 представлен график давления по поверхности аппарата в момент выхода его из контейнера, на рис. 4 - график зависимости от времени давления в газовом пузыре, образующемся с верхнего среза контейнера, в сравнении с экспериментом. На рис. 3-6 приведены распределения безразмерного давления и температуры, а также изменение коэффициента давления по безразмерному времени в газовой полости (рис. 3). Сравнение на рис. 3 и 4 при- 
ведено в виде графиков зависимостей безразмерной температуры газовой среды $T / T_{0}$ и безразмерного давления $P / P_{0}$ в задонной области в зависимости от безразмерного времени, рассчитываемого по формуле

$$
\bar{t}=t \sqrt{P_{0} / \rho_{\varkappa}} / L
$$

Здесь $T_{0}$ - начальная температура стенки; $P_{0}$ - гидростатическое давление на верхнем срезе шахты; $L$ - длина модели; $\rho_{\text {ж }}$ - плотность жидкости.

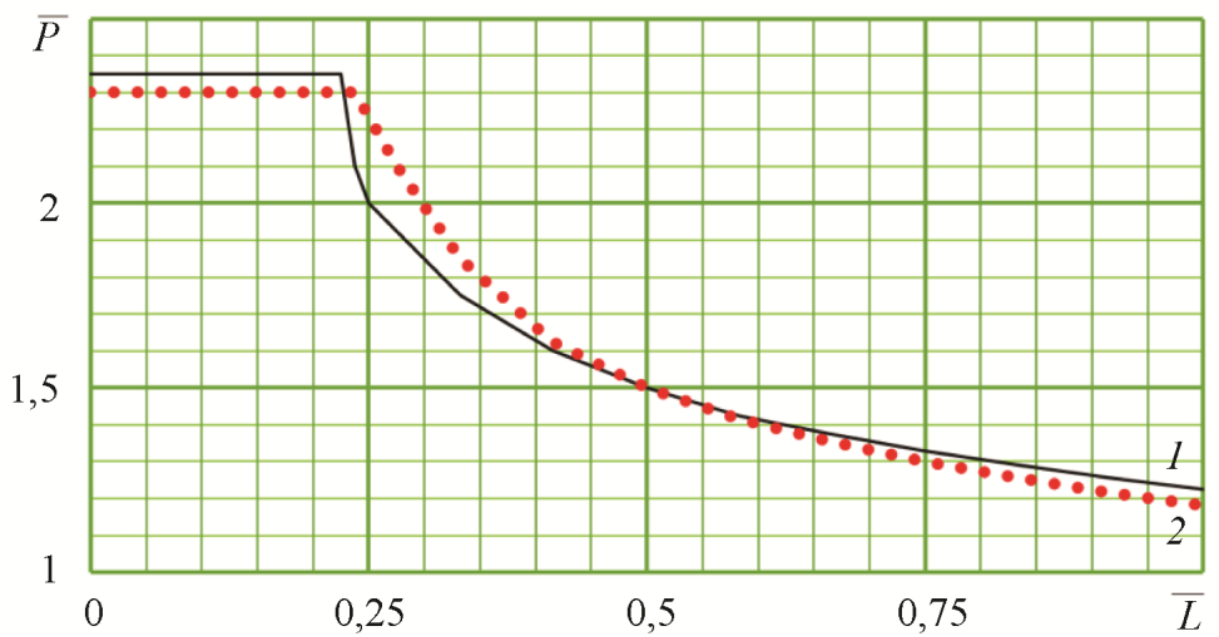

Рис. 3. Распределение давления по длине аппарата: 1 - расчет; 2 - эксперимент

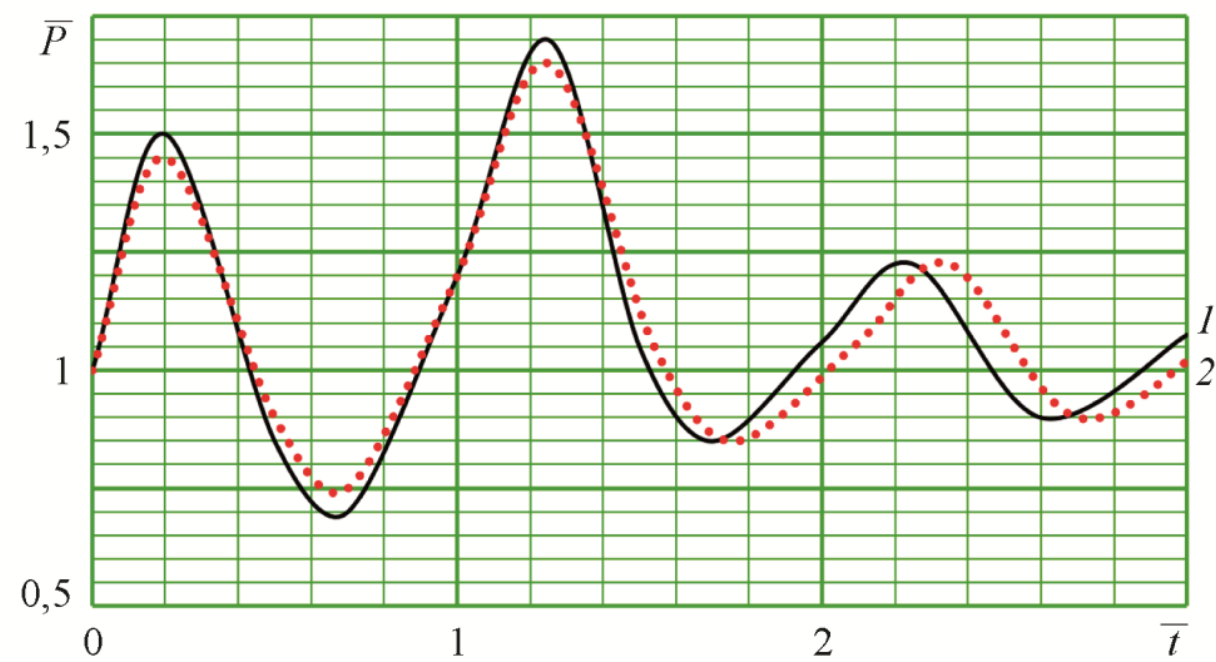

Рис. 4. Зависимость от времени давления в газовой полости: 1 - расчет; 2 - эксперимент

На рис. 5 и 6 приведены графики зависимостей от времени давления и температуры парогазовой смеси, образующейся в объеме шахты при старте аппарата на маршевом двигателе из затопленного водой контейнера [5]. На рис. 3-6 видим, что результаты имитационного моделирования удовлетворительно согласуются с экспериментальными. В работах [9-12] приведены результаты определения теплового и силового воздействия на аппараты при многофазном обтекании при больших скоростях при движении в атмосфере. 


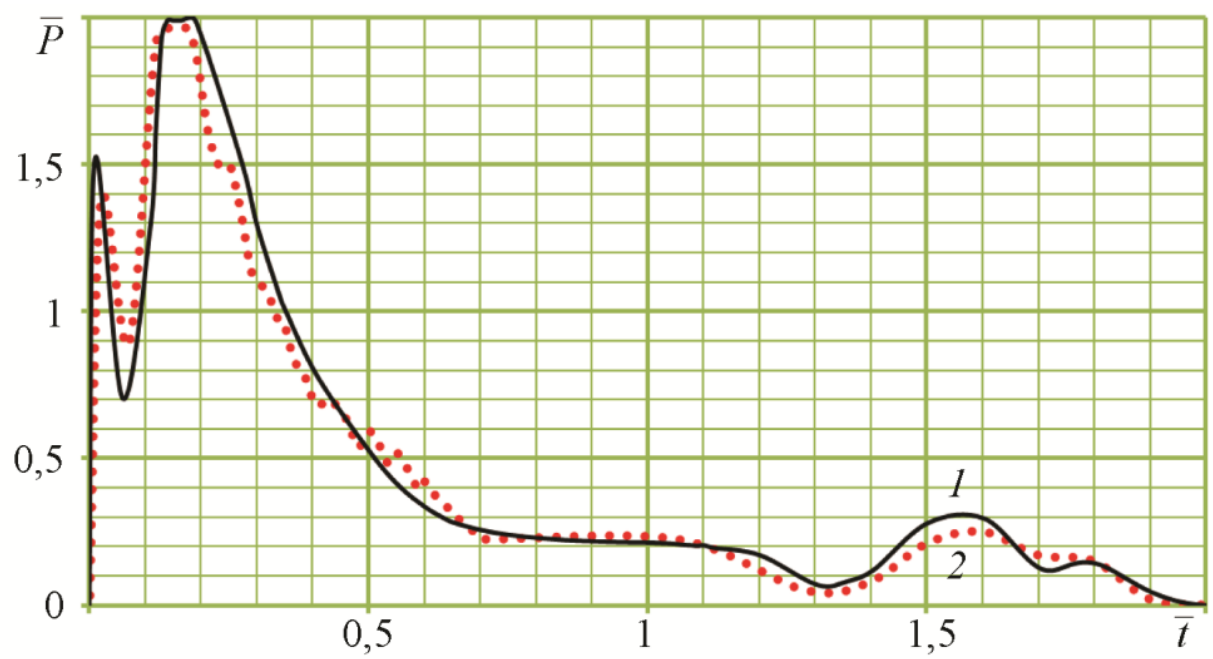

Рис. 5. Зависимость от времени давления парогазовой смеси в контейнере: 1 - расчет; 2 - эксперимент

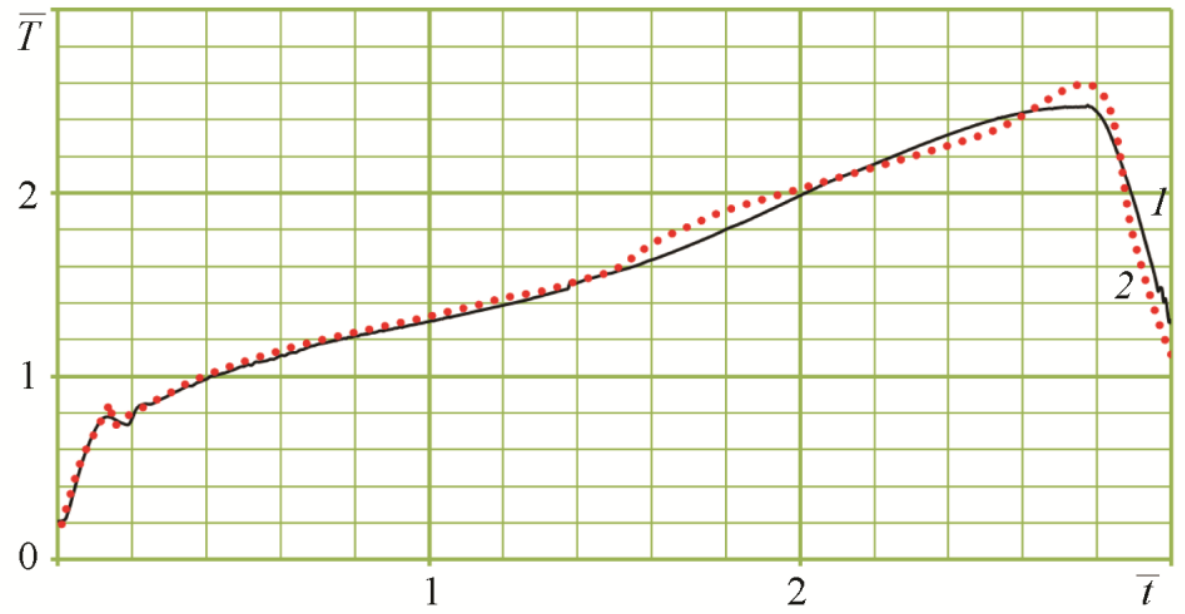

Рис. 6. Зависимость от времени температуры парогазовой смеси в контейнере:

1 - расчет; 2 - эксперимент

В целом полученные результаты позволяют сделать вывод о том, что предложенная математическая модель адекватно отражает физические процессы, протекающие при подводном старте аппарата, и может быть использована для решения задач гидродинамики по определению формы образующихся в жидкости газовых полостей и распределения давления по поверхности аппарата и носителя. Хорошее соответствие расчетных и экспериментальных данных служит подтверждением достоверности и надежности разработанного метода, который может стать основой для численного моделирования физических процессов, сопровождающих динамику автономных самоходных подводных и надводных аппаратов, обеспечивающих изучение арктического шельфа, поиска полезных ископаемых на дне Северного Ледовитого океана, а также доставку грузов в труднодоступные районы Арктики, эвакуацию людей, проведение подводных и спасательных работ.

\section{4. Заключение}

На основе метода маркеров крупных частиц разработан математический метод определения теплового и силового воздействия на аппарат при многофазном обтекании. Результаты расчетов сравниваются с экспериментальными данными. Создан программный ком- 
плекс, позволяющий проводить имитационное моделирование силового и теплового воздействия на аппарат при движении его в многофазной среде.

\section{Благодарность}

Работа выполнена при поддержке Южно-Уральского федерального научного ичентра минералогии и геоэкологии УрО РАН и АО «Государственный ракетный центр имени академика В.П. Макеева» в рамках государственного задания ЮУ ФНЦ МиГ УрО РАН по теме № 0431-2014-0001.

\section{Литература}

1. Дегтярь В. Г. Пегов В. И. Гидродинамика подводного старта ракет. - М. : Машиностроение, 2009. - 448 с.

2. Математическое моделирование процессов тепломассообмена горячих газовых струй с жидкостью при подводном старте ракет / В. Г. Дегтярь, В. И. Пегов, И. Ю. Мошкин, А. Д. Чешко // Теплофизика высоких температур. - 2019. - Т. 57, № 5. - С. 742-747.

3. Пегов В. И., Мошкин И. Ю. Расчет динамики баллистической модели // Вестник Южно-Уральского государственного университета. Серия «Математическое моделирование и программирование». - 2017. - Т. 10, № 4. - С. 56-63.

4. Дегтярь В. Г., Пегов В. И., Чешко А. Д. Исследование запуска реактивного двигателя в воде // Научно-технический вестник Поволжья. - 2016. - № 5. - С. 181-189.

5. Экспериментальное и численное моделирование стартового воздействия на подводную лодку / В. И. Пегов, А. Д. Чешко, И. Ю. Мошкин, Е. С. Меркулов // Сб. «Взгляд в будущее-2016» : труды XIV молодежной научно-технической конференции. - Санкт-Петербург : ЦКБ МТ «Рубин», 2016. - С. 598-605.

6. Пегов В. И., Мошкин И. Ю. Расчет гидродинамики кавитационного способа старта ракет // Челябинский физико-математический журнал. - 2018. - Т. 3, № 4. - С. 476-485. DOI: $10.24411 / 2500-0101-2018-13408$.

7. Численное моделирование гидродинамических нагрузок на стартующую ракету и подводную лодку / В. И. Пегов, И. Ю. Мошкин, Е. С. Меркулов, А. Д. Чешко // Вестник Концерна ПВО «Алмаз-Антей». - 2016. - № 3 (18). - С. 65-70. - ISSN 2542-054.

8. Программа для ЭВM CNC_RNSG : свидетельство о регистрации RU 2015613842 / Дегтярь В. Г., Мошкин И. Ю., Пегов В. И., Калашников С. Т., Хлыбов В. И. - № 2014619029 ; заявл. 08.09.2014; опубл. 26.03.2015.

9. Методология экспериментальных и расчетных работ по определению параметров песочно-зернистой шероховатости обгарных поверхностей / Г. Ф. Костин, В. Г. Дегтярь, С. Т. Калашников, В. Н. Савельев, В. А. Тюменцев, В. И. Хлыбов, Р. К. Швалева // Конструкции из композиционных материалов. - 2018. - № 3. - С. 29-39.

10. Методика и результаты оценки изменения теплофизических характеристик углепластика на основе фенолформальдегидного связующего при нагреве и разложении / Г. Ф. Костин, Д. А. Захарьевич, С. Т. Калашников, В. Н. Савельев, Р. К. Швалева // Конструкции из композиционных материалов. - 2018. - № 4. - С. 63-70.

11. Калашников С. Т., Мокин Ю. А., Швалева Р. К. Расчетно-экспериментальное исследование изменения формы малых искажений боковой поверхности в процессе абляции углепластикового теплозащитного покрытия модели спускаемого летательного аппарата // Конструкции из композиционных материалов. - 2018. - Вып. 4 (152). - С. 42-47.

12. Мокин Ю. А., Калашников С. Т., Швалева Р. К. Оценка дисперсии коэффициента возмущающей аэродинамической нормальной силы тела вращения с малыми случайными искажениями поверхности композитного теплозащитного покрытия при сверхзвуковом обтекании // Конструкции из композиционных материалов. - 2020. - № 3. - С. 3-8. 